\title{
A Functional Precision Oncology Approach to Identify Treatment Strategies for Myxofibrosarcoma Patients
}

\author{
Chantal Pauli', Lamberto De Boni ${ }^{2}$, Jonathan E. Pauwels ${ }^{3}$, Yanjiang Chen', Lara Planas-Paz ${ }^{1}$, Reid Shaw ${ }^{4}$, \\ Brooke M. Emerling ${ }^{5}$, Carla Grandori ${ }^{4}$, Benjamin D. Hopkins ${ }^{2}$, and Mark A. Rubin ${ }^{3,6}$
}

\section{ABSTRACT}

In this era of precision medicine, numerous workflows for the targeting of high-recurrent mutations in common tumor types have been developed, leaving patients with rare diseases with few options. Here, we implement a functional precision oncology approach utilizing comprehensive genomic profiling in combination with high-throughput drug screening, to identify tumor-specific drug sensitivities for patients with rare tumor types such as myxofibrosarcoma. From a patient with a high-grade myxofibrosarcoma, who was enrolled in the Englander Institute for Precision Medicine (EIPM) program, we established patient-derived 3D sarco-spheres and xenograft models for functional testing. In the absence of a large cohort of clinically similar cases, high-throughput drug screening was performed on the patient-derived cells, and compared with two other myxofibrosarcoma lines and a benign fibroblast line to functionally identify tumor-specific drug sensitivities. The addition

\section{Introduction}

Soft tissue sarcomas arise from cells of mesenchymal origin and represent extremely diverse neoplasms with over 70 histologic subtypes (1). Sarcomas account for less than $1 \%$ of all cancers, and have significant levels of phenotypic and genomic diversity, which complicate their diagnosis and make it difficult to identify effective treatments. Myxofibrosarcoma (MFS), formerly known as MFH (malignant fibrous histiocytoma) myxoid variant, is a soft tissue sarcoma usually located in the extremities of elderly patients. Tumor grades vary and tumor growth is characterized by a multinodular growth pattern with spindle to polygonal sarcoma cells embedded

\begin{abstract}
'Department of Pathology and Molecular Pathology, University Hospital Zurich, Zurich, Switzerland. ${ }^{2}$ Department of Genetics and Genomic Sciences, Icahn School of Medicine at Mount Sinai, New York, New York. ${ }^{3}$ Englander Institute for Precision Medicine, Weill Cornell Medicine-New York Presbyterian Hospital. New York, New York. ${ }^{4}$ SEngine Precision Medicine, Seattle, Washington. ${ }^{5}$ Cancer Metabolism and Signaling Networks, Sanford Burnham Prebys Medical Discovery Institute, La Jolla, California. ${ }^{6}$ Department for BioMedical Research, Bern, Switzerland.
\end{abstract}

Note: Supplementary data for this article are available at Molecular Cancer Research Online (http://mcr.aacrjournals.org/).

B.D. Hopkins and M.A. Rubin contributed equally and are co-last authors of this article.

Corresponding Author: Chantal Pauli, Department of Pathology and Molecular Pathology, University Hospital Zurich, Zurich 8091, Switzerland. Phone: 4179453-4416; E-mail: chantal.pauli@usz.ch

Mol Cancer Res 2021;XX:XX-XX

doi: 10.1158/1541-7786.MCR-21-0255

This open access article is distributed under Creative Commons AttributionNonCommercial-NoDerivatives License 4.0 International (CC BY-NC-ND).

(C)2021 The Authors; Published by the American Association for Cancer Research of functional drug sensitivity testing to complement genomic profiling identified multiple therapeutic options that were further validated in patient derived xenograft models. Genomic analyses detected the frequently known codeletion of the tumor suppressors $C D K N 2 A / B$ together with the methylthioadenosine phosphorylase (MTAP) and a TP53 E286fs*50 mutation. High-throughput drug screening demonstrated tumor-specific sensitivity to compounds targeting the cell cycle. Based on genomic analysis and highthroughput drug screening, we show that targeting the cell cycle in these tumors is a powerful approach.

Implications: This study demonstrates the potential of functional testing to aid clinical decision making for patients with rare or molecularly complex malignancies when combined with comprehensive genomic profiling.

within a variable myxoid stroma containing long curvilinear vessels $(2,3)$. The standard of care for primary MFS is surgical resection and radiotherapy; however, increased tumor grades and stages are frequently observed after local recurrence (4). The overall survival rate is approximately $70 \%$ at 5 years. However, $30 \%$ to $40 \%$ of these patients have metastatic disease that is treated with chemotherapy and the disease eventually progresses leading to disease-related death $(5,6)$. Due to their heterogeneity and complex karyotypes, it is difficult to isolate the molecular pathogenesis of MFS, thus limiting the use of targeted therapeutic strategies. Previous molecular studies using comprehensive sequencing efforts have described common aberrations with other related sarcoma subtypes with highly complex karyotypes, such as undifferentiated pleomorphic sarcoma (UPS). To date, the most significant alterations described in MFS, are located in tumor suppressor genes such as TP53, RB1, CDKN2A, CDKN2B, and NF1 (7-15). Previous work has investigated potential drug targets but at present no effective targeted therapies are available for patients with MFS especially in the metastatic setting (14).

Precision medicine approaches have been effectively employed in related genetically simple soft tissue tumors, for example, gastrointestinal stromal tumors (GIST) where imatinib and sunitinib were identified as effective therapies (16) or more recently in spindle cell tumors with a NTRK-rearrangement (e.g., infantile fibrosarcoma; ref. 17). Here, we leverage a novel functional precision oncology approach that integrates genomic profiling, high-throughput drug screening, and in vivo validation of patient-derived xenografts to identify and validate effective therapeutic options for patients with high-grade MFS.

\section{Materials and Methods}

\section{Specimen procurement}

Fresh tissue was harvested from a resection specimen, according to Institutional Review Board (IRB)-approved protocols, from a patient 
(WCM197) suffering from a myxofibrosarcoma that got enrolled in the Precision Medicine Clinic at Weill Cornell Medicine and New York-Presbyterian Hospital (WCMC-NYPH). Written informed consent from the patient was obtained in accordance with recognized ethical guidelines (e.g., Declaration of Helsinki, CIOMS, Belmont Report, U.S. Common Rule), and the study was approved by an IRB.

\section{Tissue sample processing}

For next-generation sequencing, the Maxwell 16 Tissue DNA Purification Kit (Promega) was used to extract DNA. DNA preparation, sequencing, and computational analysis were performed as previously described $(18,19)$.

\section{In vitro models}

A 3D sarco-sphere model with a corresponding monolayer cell strain was established from a myxofibrosarcoma resection specimen (WCM197). The OH931 cells were kindly provided by Dr. J. Bridge, Nebraska Medical Center, Omaha, NE, and the NMFH-1 cell line was obtained via the REDKEN tissue bank at the University of Tokyo, Japan, upon the kind approval from Dr. Ogose $(20,21)$. The Human Foreskin Fibroblast (HFF) cells were provided by Dr. Carla Grandori, SEngine Precision Medicine, Seattle, WA, as a control specimen for high throughput drug screening (22). All three sarcoma cells (WCM197, OH931, NMFH-1) were sequenced using the comprehensive genomic profiling platform FoundationOne in the Laboratory from the University Hospital in Zurich for validation. All cell strains and cell lines used in this work were maintained in RPMI (Invitrogen) with $10 \%$ FBS (Denville), $100 \mathrm{U} / \mathrm{mL}$ penicillin and $100 \mu \mathrm{g} / \mathrm{mL}$ streptomycin (Gibco). For the 3D sarco-sphere model, cells were plated in individual Matrigel drops (growth factor reduced, Corning) $5 \mu \mathrm{L}$. Drops were plated in suspension plates, after they solidified the plates were filled with media and kept in a cell culture incubator at $37^{\circ} \mathrm{C}$ and $5 \% \mathrm{CO}_{2}$. Twenty-four hours after plating, the individual Matrigel drops were mechanically detached from the plate and the sarcospheres were grown in suspension.

\section{High-throughput drug screening}

Each step was done by laboratory automation, that is, cell plating, drug addition, and plate reading. A range of 900 to 2,000 cells (a value determined by optimization studies for doubling time for each cell type) were plated in 384-well plates on day one and treated with six different drug concentrations 24 hours after plating. Drug concentrations ranged from $10 \mu \mathrm{mol} / \mathrm{L}$ to $33 \mathrm{pmol} / \mathrm{L}$. Cell viability was measured at 6 days following the drug treatment using Cell-Titer Glo 2.0 (Promega). Cell-Titer Glo 2.0 was selected to assess cell viability as it was previously determined to exhibit the broadest linear range and minimally affected by the time necessary to process multiple plates. A Biotek Synergy H4 plate reader was used to read the luminescence signal. Raw luminescence obtained from each well was normalized to the average of multiple DMSO control wells to be considered $100 \%$ viability (maximal DMSO concentration used was $0.1 \%$ ). Mitomycin C and/or bortezomib were used as positive controls, as these are drugs with high toxicity in a majority of cell types. Dose response curves were generated for each drug and area under the curve (AUC) and $\mathrm{IC}_{50}$ were calculated for each drug as described (23). Drug Library: The 395compound library was purchased from Selleck Chemicals and targets a broad range of cancer-related pathways (Supplementary Table S1). The drug library contains FDA-approved and tool compounds that target a broad range of oncogenic processes, including PI3K, HDAC, mTOR, CDK, JAK, and RTK. Drugs were diluted to an 8-point dose curve incorporating a 3 -fold dilution step. The highest final drug concentrations were $5 \mu \mathrm{mol} / \mathrm{L}$; DMSO or PBS remained consistent across the wells at $0.05 \%$. For drug combinations, the IC30 was used as a sensitizer drug combined with the entire library as previously published (23). For manual validation the same sensitizing concentration (IC30) was used as with the high-throughput screen.

\section{In vitro drug validation}

For monolayer drug validation, 2,000 cells were plated in triplicates in 96-well plates for monolayer drug testing. Twenty-four hours after plating, drugs were tested in a 6-point dilution dose response log scale to determine the $\mathrm{IC}_{50}$ values, the highest dose was $10 \mu \mathrm{mol} / \mathrm{L}$ and the lowest dose was $4.7 \mathrm{pmol} / \mathrm{L}$ with an assay time of 96 hours. We used Cell-Titer Glo 2.0 and CyQuant NF Cell Proliferation Assay (Promega) for the endpoint read-out in monolayer culture. Plates were read using the Spectra Max L (Molecular Devices) at $570 \mathrm{~nm}$. Analysis was performed running a nonlinear regression (curve fit) method in Prism 6 for Mac OS X. For 3D drug validation, up to ten $5 \mu \mathrm{L}(500$ cells $/ \mu \mathrm{L})$ Matrigel:cell suspension mix were plated in a 6-well suspension plate (SARSTEDT Ltd). Twenty-four hours later, drugs were added. Drug combinations were done with one drug at the IC30 and the other drug in a 6-point dilution dose response log scale to determine the $\mathrm{IC}_{50}$ values, the highest dose was $10 \mu \mathrm{mol} / \mathrm{L}$ and the lowest dose was $4.7 \mathrm{pmol} / \mathrm{L}$ with an assay time of 96 hours. For sarco-sphere imaging, they were incubated up to one hour with the CyQuant NF Cell Proliferation Assay. Pictures were continuously taken over several days using a X-cite series 120Q UV-lamp and an Olympus CKX41 microscope.

\section{Western blotting}

Lysates were prepared in $1 \times$ CST Lysis Buffer. Protein concentration was evaluated with the BCSA Kit (Pierce). Lysates were run out on a $4 \%$ to $20 \%$ Tris-Glycerin Gels (Thermo Fisher Scientific). Primary antibodies against CDKN2A (1:2000, Abcam Cat\# ab208349), MTAP (1:2000, Abcam Cat\# ab126623), RB (1:1000, Abcam Cat\# ab32513), Cleaved Caspase-3 (Asp 175; 1:1,000, Cell Signaling Technology, Cat\# 9664), cleaved PAPR (aSP214; 1:1,000, Cell Signaling Technology, Cat\# 32563), and Actin (1:2,000, EMD Millipore clone 4, Cat\# 1501) were incubated overnight at $4{ }^{\circ} \mathrm{C}$ in $5 \%$ bovine serum albumin. Following three washes with TBS-T, the blot was incubated with horseradish peroxidase-conjugated secondary antibody and immune complexes were visualized by enhanced chemiluminescence detection (ECL Plus Kit, Pierce).

\section{Xenograft studies}

Animal procedures were approved by the IACUC protocol 20130016. For xenograft development, $1 \times 10^{6} \mathrm{WCM} 197$ cells were mixed with Matrigel $1: 1(\mathrm{v} / \mathrm{v})$ in a total volume of $100 \mu \mathrm{L}$ and injected subcutaneously in the lower abdomen from nude mice (The Jackson Laboratory). For in vivo drug studies, 5 mice were tested per treatment $\operatorname{arm}(N=5)$, for each of four independent experiments. Treatment was initiated after tumors reached a diameter of approximately $0.65 \mathrm{~cm}$, typically 8 days postinjection. The following drugs and dosing schedules were used: abemaciclib: low-dose $45 \mathrm{mg} / \mathrm{kg}$ and high-dose $90 \mathrm{mg} / \mathrm{kg}$ diluted in 1\% HEC/PBS ( $\mathrm{pH} 2$ ), oral gavage daily, pralatrexate: $35 \mathrm{mg} / \mathrm{kg}$ diluted in 10\% DMSO and 60\% PEC solution day $(1,4$, $11,14,21,24$ i.p), Leucovorin was given $50 \mathrm{mg} / \mathrm{kg}$ diluted in $0.9 \%$ sterile $\mathrm{NaCl}$ intraperitoneally the 2 following days after pralatrexate injections (days $2,3,5,6,12,13,22,23,25,26$ ), volasertib $2 \mathrm{mg} / \mathrm{kg}$ diluted in $10 \%$ DMSO with $30 \%$ PEC intraperitoneally once a week. Body weight was taken once per week. Tumor size was evaluated by caliper three times a week. Volumes were calculated using the formula $4 / 3 \pi^{*}\left(\left(\operatorname{sqrt}\left(L^{*} W\right)\right) / 2\right) 3$, where $L$ is the minor tumor axis and $W$ is the 
major tumor axis. Tumors were harvested when their diameter reached a size $>1.5 \mathrm{~cm}$ at which point portions were snap frozen and fixed for 24 hours with Paraformaldehyde Solution (4\% in PBS, Affymetrix) for paraffin embedding and sectioning.

\section{Immunohistochemistry}

Xenograft tumor sections $(2 \mu \mathrm{m})$ were antigen retrieved with $10 \mathrm{mmol} / \mathrm{L}$ citrate acid, $0.05 \%$ Tween 20 , pH6.0), and stained using a Bond III automated immunostainer (Leica Microsystems). Antibodies targeting Ki67 (1:500; Abcam Cat\# ab16667) 1:500 and cleaved caspase-3 (Asp175;5A1E; 1:200; Cell Signaling Technology, Cat\# 9664) were used.

\section{Data availability statement}

Raw data for this study were generated at the Englander Institute for Precision Medicine and the tumor profiling laboratory in the Department for Pathology and Molecular Pathology, University Hospital Zurich. Derived data supporting the findings of this study are available from the corresponding author upon request.

\section{Results}

CDKN2A/CDKN2B and MTAP codeletion - targeting a common alteration in a subgroup of patients with high-grade MFS

A senior female patient presenting with a swelling on her medial left thigh was enrolled into the precision medicine clinic at Weill Cornell Medicine in New York. The patient underwent tumor excision and was diagnosed with a high-grade MFS. Fresh tissue was harvested for the EXaCT-1 (whole-exome sequencing) test and ex vivo model development. Histological analysis showed a high-grade MFS and wholeexome sequencing identified a common homozygous codeletion of the tumor suppressors $C D K N 2 A / C D K N 2 B$ and the methylthioadenosine phosphorylase (MTAP) beside a TP53 E286fs*50 mutation. We established a novel 3D sarco-sphere model (WCM197) and the corresponding monolayer cell strain from this sarcoma. These models were used for functional testing (Fig. 1A-E). Drug sensitivity of the patient-derived cells (WCM197) was compared with two previously established and published MFS lines (OH931, NMHF-1) and a primary human fibroblast line (HFF) as a normal control. $C D K N 2 A / B / M T A P$ and $R B 1$ status was confirmed in all the cell lines by comprehensive genomic profiling (FoundationOneHeme). Comprehensive genomic profiles are listed in the supplements (Supplementary Table S2). OH931 and WCM197 share RB1 wild-type and $C D K N 2 A / B / M T A P$-null status, while NMFH-1 was confirmed as RB1 and CDKN2A/B/MTAP wild type. The simultaneous loss of the tumor suppressors CDKN2A/MTAP and MTAP was therapeutically targeted and we found that the $C D K N 2 A / B$ / $M T A P$-null cells (WCM197, OH931) were highly sensitive to a combination with the CDK4/6-inhibitor abemaciclib, a third line selective CDK4/6-inhibitor and the antifolate, pralatrexate, which has been shown to inhibit de novo purine biosynthesis (Fig. 2A and B). In the WCM197 and OH931 (CDKN2A/B and MTAP-null) cell models, a combination of abemaciclib and pralatrexate showed increased cell death compared with abemaciclib or pralatrexate as a single agent (Fig. 2C-F).

\section{Personalized high-throughput drug screening nominates potential drug targets of response}

All three MFS lines (WCM197, OH931, NMFH-1) and the normal control line (HFF) were subjected to high throughput drug screening using a customized Selleckchem library containing 386 drugs, includ- ing FDA-approved and tool compounds targeting a broad range of oncogenic processes, including PI3K, HDAC, mTOR, CDK, JAK, and RTK (Supplementary Table S1). These broad drug screens demonstrated resistance of all three MFS lines to the majority of targeted agents in the library as compared to the HFFs. The tumor cells did show moderate sensitivity to a number of microtubule-inhibiting chemotherapies such as docetaxel, paclitaxel, vinblastine, and vincristine. More interesting was the sensitivity of WCM197 and OH931 (CDKN2A/B, MTAP-null, RB1 wild type) to methotrexate and pralatrexate and the higher sensitivity to the CDK4/6 inhibitor abemaciclib as compared with NMFH-1 and HFF (CDKN2A/B, MTAP, and RB1 wild type), demonstrating a selectivity of these therapies for these tumors (Fig. 3A and B). We also observed a selective sensitivity of the CDKN2A/B/MTAP mutant lines to polo-like kinase 1 (PLK1) inhibition. The relative sensitivity of these lines to PLK inhibitors volasertib and rigosertib was validated in the 3D sarco-sphere model. The induction of apoptosis in the tumor cells by volasertib was demonstrated ex vivo via western blot for cleaved caspase- 3 and cleaved PARP (Fig. 3C-F).

\section{In vivo validation of drug efficacy}

We assessed the in vivo efficacy of the identified therapeutics abemaciclib with pralatrexate and volasertib in mice bearing PDX tumors from WCM197. To generate the xenografts, half a million cells from this patient's tumor, were engrafted $\left(0.5 \times 10^{6}\right)$ in the lower abdominal region of nude mice. The selective CDK4/6 inhibitor abemaciclib, and the antifolate pralatrexate, either administered as single agents or in combination were compared to vehicle and tumor growth was assessed. Both agents used as monotherapies showed reduced tumor growth, pralatrexate being more effective as a monotherapy for suppressing tumor growth in the PDX model. The combination of the two compounds was able to induce stable disease in these highly aggressive xenografts without noticeable toxicity (as gauged by weight loss; Fig. 4A and B). All mice treated with pralatrexate received leucovorin to reduce potential toxic effects of the antifolate pralatrexate that have been noticed in other mouse studies and is a common supplement in humans receiving pralatrexate or related therapies. Immunohistochemical analysis showed a marked reduction in the number of proliferating cells as indicated by Ki67 staining of the treated tumors, especially those from the mice receiving the combination of abemaciclib and pralatrexate (Fig. 4C). Reciprocally, the tumors from these mice showed increased levels of apoptosis as indicated by staining for cleaved caspase-3 (Fig. 4D).

Considering the elevated age of the patient population suffering from these tumors that abemaciclib is a well-tolerated drug, we further investigated a dose dependent monotherapy approach with CDK4/6 inhibitor (24). We tested low ( $45 \mathrm{mg} / \mathrm{kg}$ ) versus high $(90 \mathrm{mg} / \mathrm{kg})$ dose daily in an experiment together with volasertib (PLK1-inhibitor) once a week. Using abemaciclib at a higher dose once daily as a single agent we were able to prolong survival and retard tumor growth without any noticeable toxicity in the animals as gauged by behavior or weight loss (Fig. 4E and F). Volasertib dosed once weekly as monotherapy was also well tolerated and reduced tumor growth. Taken together, these results show the beneficial effect of targeting the cell cycle in a model of myxofibrosarcoma and highlight a potential new venue of treatment for this rare tumor type.

\section{Discussion}

The complex genomics of MFS, have made the identification of effective targeted therapeutics for patients who progress after 
A

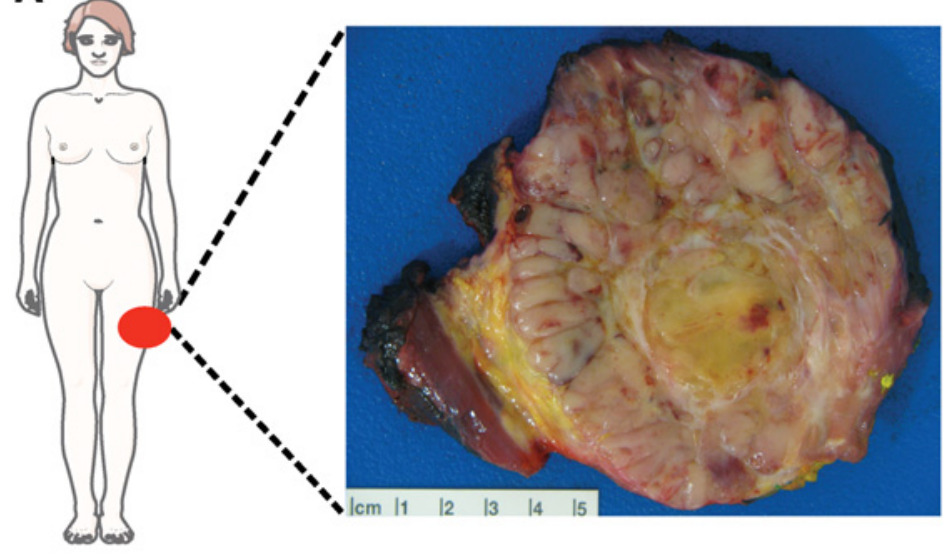

C

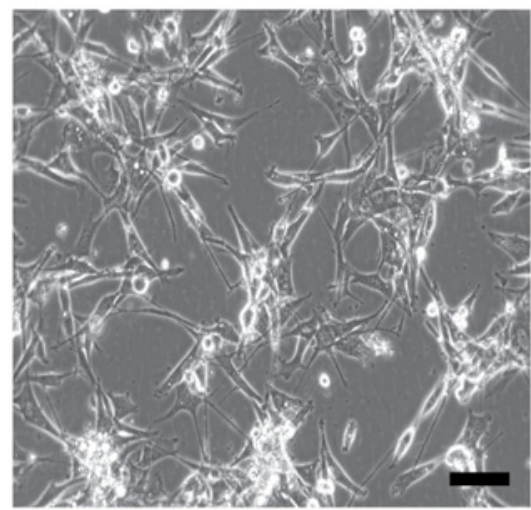

B
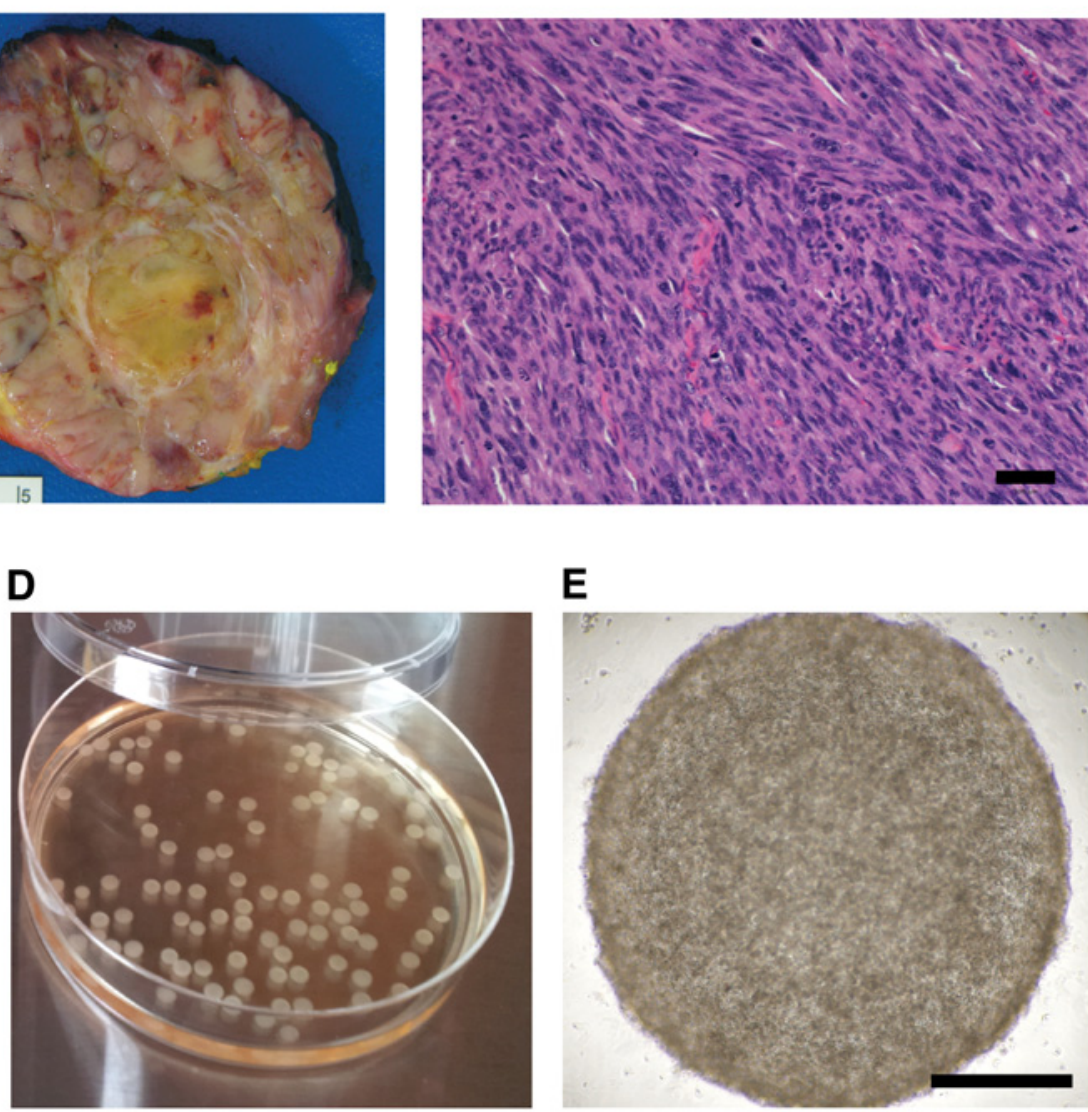

E

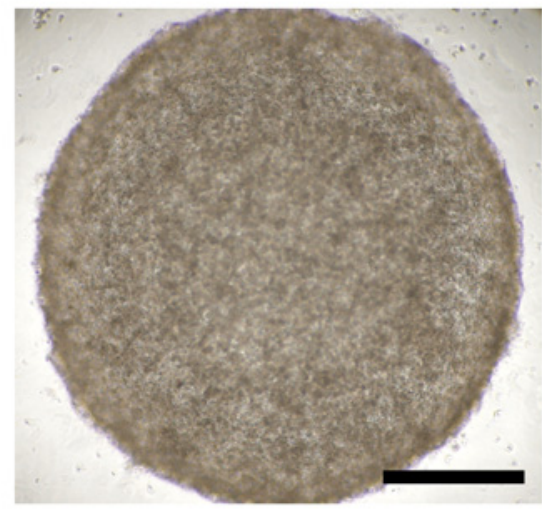

Figure 1.

Preclinical functional precision cancer model of a myxofibrosarcoma patient. A and B, Elderly female patient with a mass in her left thigh. Gross examination showed a soft tissue tumor and the histology was compatible with a high grade myxofibrosarcoma (scale bar, $100 \mu \mathrm{m}$ ). A novel patient derived 3D sarco - sphere model for functional testing (high throughput drug screening) and in vivo modeling was established. C, Shows cells grown in monolayer, scale bar, $20 \mu \mathrm{m}$ and the 3D sarcosphere model, scale bar, $1 \mathrm{~mm}$ ( $\mathbf{D}$ and $\mathbf{E}$ ).

surgical resection and radiation therapy challenging. Using our functional precision oncology platform $(18,23)$, that includes nextgeneration sequencing, high-throughput drug screening, and validation in patient-derived models, we demonstrate the potential clinical utility of this precision medicine approach for patients with rare tumor types. In this instance through our pipeline, we were able to identify multiple therapeutic options for the treatment of this tumor.

Deletions of CDKN2A,CDKN2B, and MTAP genes frequently cooccur due to their physical proximity on $9 \mathrm{p}$ (25), and account for approximately $15 \%$ of MFS according to the TCGA and other published studies (13). As one of our patients showed this codeletion, we functionally investigated the $G_{1}$ checkpoint as a potential treatment approach. Drug screening and validation of patient derived models independently demonstrated that cells from this tumor had enhanced sensitivity to compounds which target the cell cycle. Specifically, selective CDK4/6 inhibitors, such as abemaciclib and antifolates such as pralatrexate as well as PLK-1 inhibitors, such as volasertib were identified by high-throughput drug screening. As the cell cycle is one of the hallmarks of cancer and seems to be altered rather frequently in sarcoma, we considered the cell cycle a reasonable target for novel treatment strategies. Abemaciclib has a very high selectivity for CDK4 and CDK6 and a safety profile that allows for continuous dosing, resulting in sustained target inhibition (24) with a low toxicity profile. Of note, this tumor had no RB1 alterations (13), which is crucial in order for CDK4/6 inhibitors to be effective. Cells with loss of MTAP function cannot cleave endogenous methyl-thioadenosine to adenine and 5-methylthioribose-1-phosphate, a precursor of methionine, and, therefore show enhanced sensitivity to inhibitors of de novo purine biosynthesis, such as pralatrexate an antimetabolite (26). We demonstrate that MFS cells and tumors with $C D K N 2 A / B$ and MTAP loss and $R B 1$ wild type status show high sensitivity to these drugs in combination. The use of single agent palbociclib as the most advanced CDK4/6 inhibitor has been preclinically and clinically tested in different soft tissue tumor types such as uterine leiomyosarcoma, dedifferentiated liposarcoma, and chordoma $(27,28)$. CDK4/6 inhibitors mainly have a cytostatic effect. Their use as a single agent has led to prolonged disease stabilization (PFS) at 12 weeks for palbociclib in $57.2 \%$ (NCT01209598) and for abemaciclib in 76\% (NCT02846987) of patients with well- and de-dedifferentiated liposarcoma. There is an unmet need for more effective combination treatments as these have been shown to be more promising (29). Recently, dual targeting of CDK4/6 inhibitors and IGF-1R inhibitors was shown to have synergistic effects in patients with Ewing sarcoma (30). In our case, the combination of drugs was guided by the functional high-throughput 
A Abemaciclib

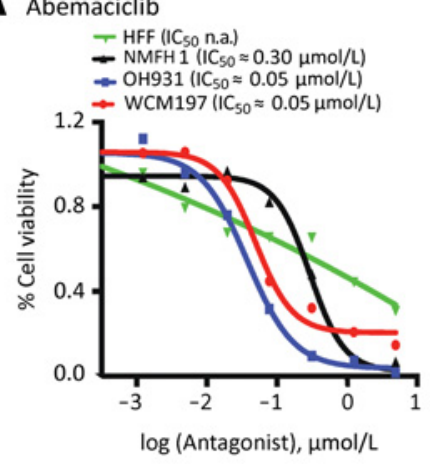

D

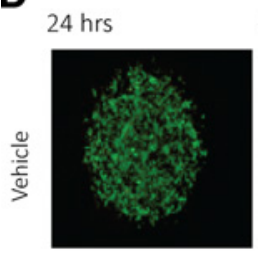

48 hrs
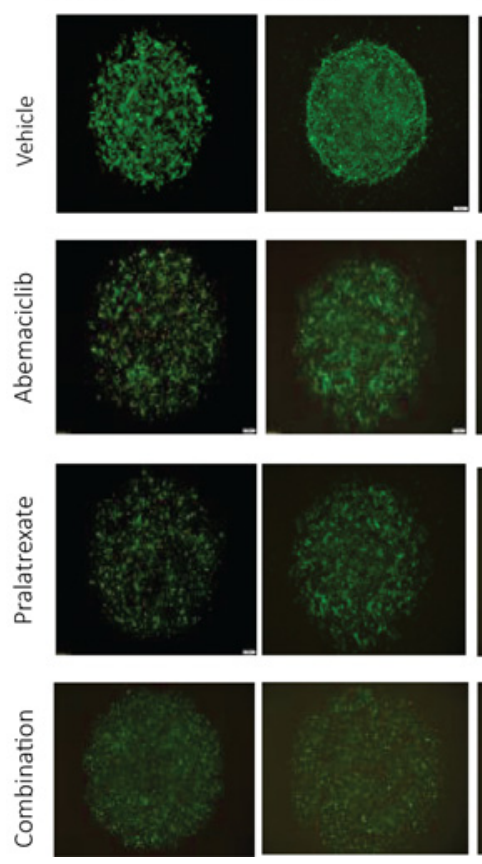

B

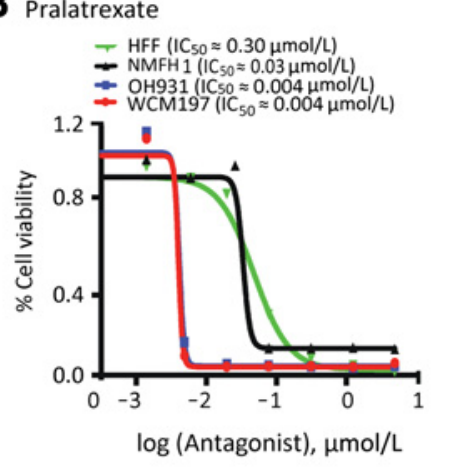

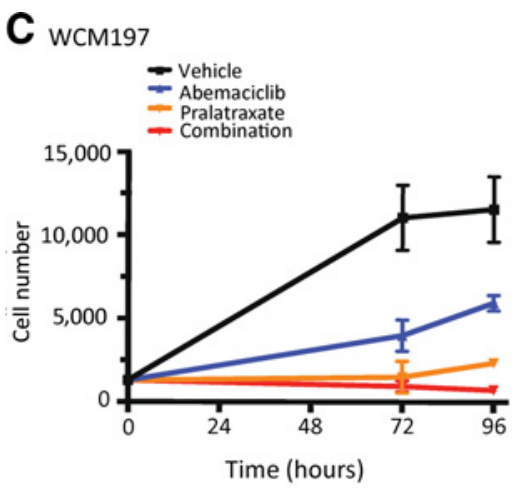

E

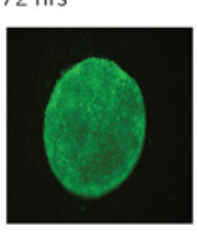

96 hrs
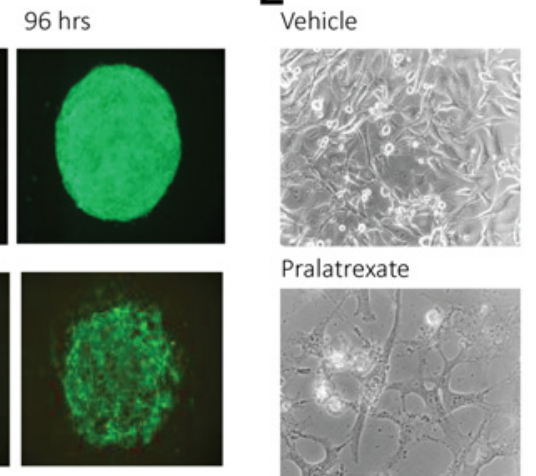

Pralatrexate

Abemaciclib

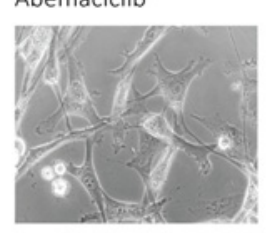

Combination
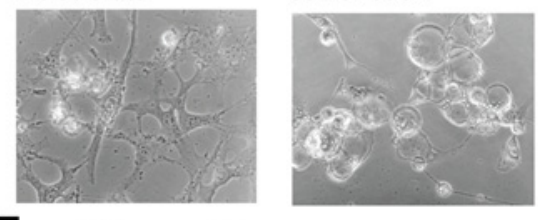

$\mathbf{F}$
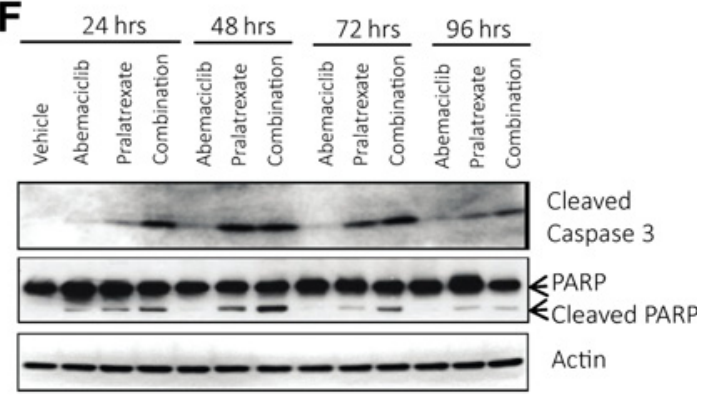

\section{Figure 2.}

A and B, Ex vivo drug testing of the novel patient-derived cell model (WCM197) together with the OH931, NMFH-1, and HFF (benign human fibroblasts) using abemaciclib, a second-line CDK4/6 inhibitor and pralatrexate, an antifolate inhibitor in a 6- to 8-point dilution dose response log scale to determine the IC ${ }_{50}$ values, highest dose was $10 \mu \mathrm{mol} / \mathrm{L}$ and lowest dose was $4.7 \mathrm{pmol} / \mathrm{L}$ with an assay time of 96 hours. Greater cytostatic and cytotoxic effect was seen in the cells with the CDKN2A/B and MTAP-null status (WCM197 and OH931) compared with CDKN2A/B and MTAP wild-type status (NMFH-1 and HFF). C, The combination of pralatrexate (IC30) and abemaciclib killed all our patient cells within 48 hours compared with the vehicle and abemaciclib and pralatrexate monotherapy. D, WCM197 sarco-sphere formation is completely inhibited with the combination of abemaciclib and pralatrexate. E, Drug combination of abemaciclib and pralatrexate causes massive cell swellings and cytoplasmatic vacuole formation. F, Cleaved caspase-3 and cleaved PARP at 24,48 , and 72 hours were detected with the combination of abemaciclib and pralatrexate, indicating the induction of cell death in the tumor cells after treatment.

drug screen and complemented by molecular profiling for drug validation. CDK4/6 inhibitors are causing cell-cycle arrest in the $G_{1}$ phase, while antimetabolites such as pralatrexate, primarily act in the S-phase of the cell cycle and induce apoptosis, which we also confirmed by Western blot in our cell model. Using this drug combination, we targeted the $G_{1}$ and $S$ phase of these cells and induced cell death.

The serine/threonine kinases Polo-like kinase (PLK) 1 is a key regulator of cell cycle with the main functions in the transition from $\mathrm{G}_{2}$ to $\mathrm{M}$-phase, the regulation of the mitotic entry and the coordination of centrosomes as well as spindle assembly, chromosome segregation, and entry into mitosis (31). Cells exposed to PLK1 inhibitors are unable to establish a bipolar spindle and cannot align chromosomes in the metaphase plate, which in turns activates cell-cycle checkpoints, leading to a mitotic arrest and eventually cell death. This vulnerability of MFS to a major regulator of the mitotic spindle is also consistent with the finding that the only active chemotherapies detected in the broad high-throughput drug screen, taxanes, and vinca alkaloids were all related to microtubule function. Inhibitors of PLK1 have been previously explored in cell lines of different sarcoma type as monotherapy or in combination in a preclinical setting with promising results $(25,29,30)$ but clinical trial experience is still minimal. While volasertib was recognized as "innovative therapy for leukemia" and granted orphan drug designation by the FDA, recent studies in animal models indicate cardiovascular toxicity associated with PLK1 
Pauli et al.

A

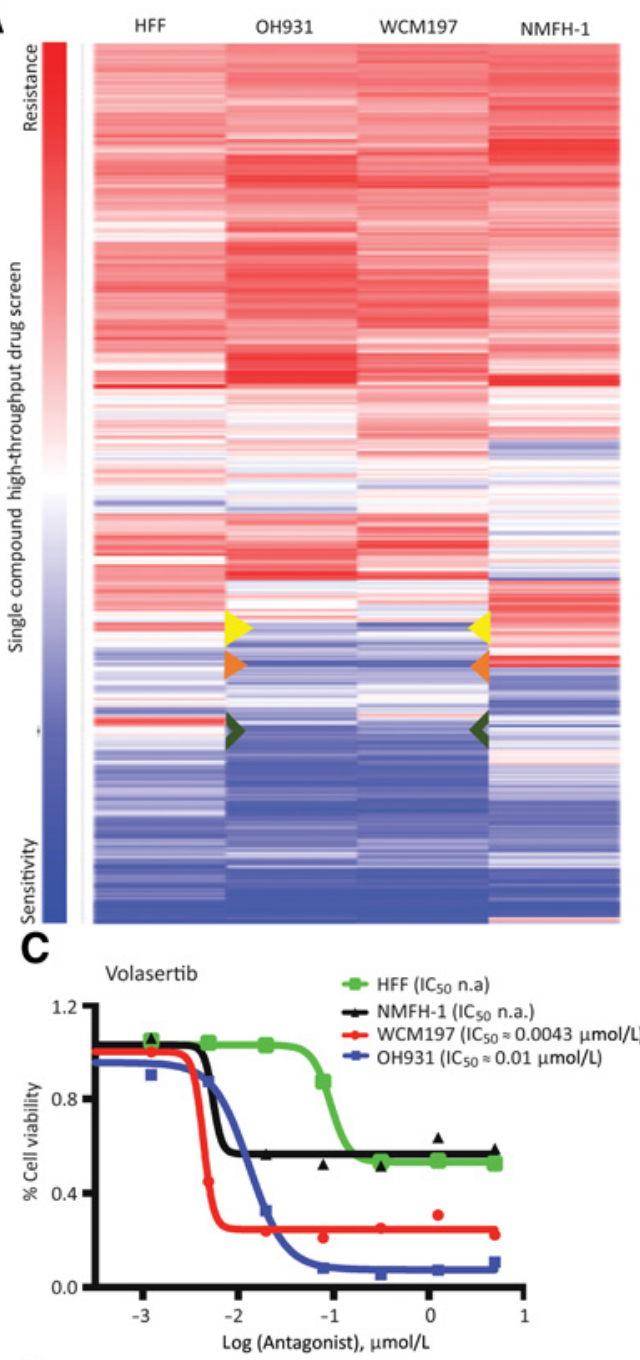

E

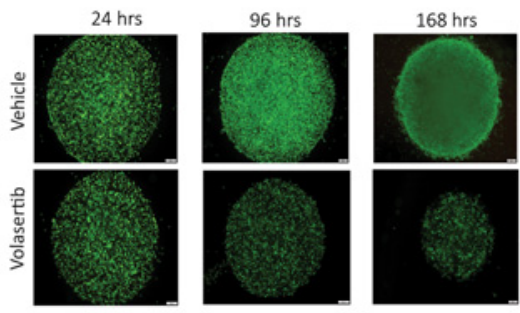

B
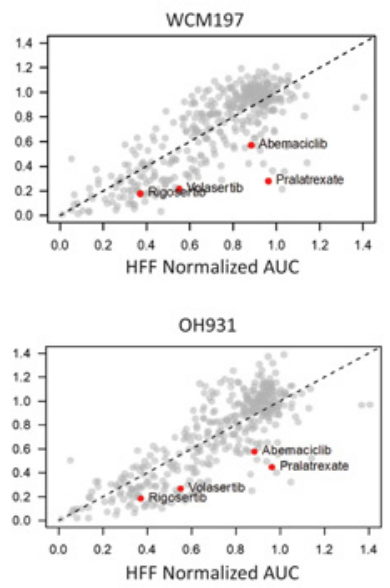

NMFH-1

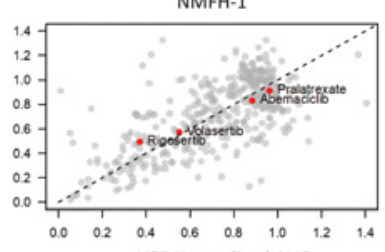

HFF Normalized AUC

\section{D}

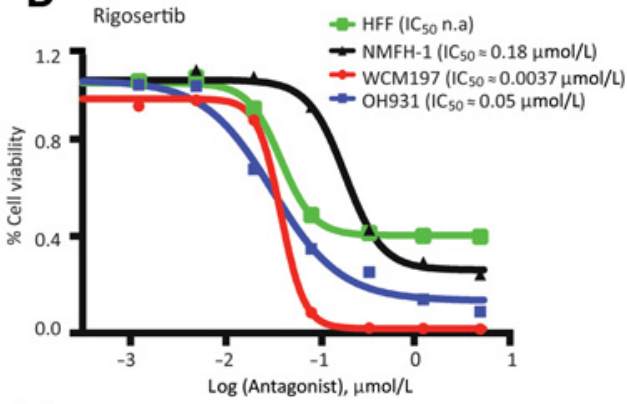

$\mathbf{F}$

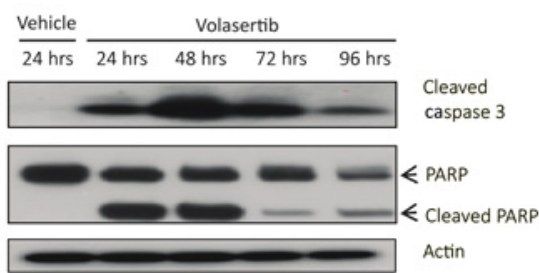

Figure 3.

A, High-throughput drug screening of all 4 cell strains (HFF, OH931, WCM197, NMFH-1) shown as a heatmap (blue is sensitive to red resistant). RB1 wt and CDKN2A/B/ MTAP null cells (WCM197 and OH931) show higher sensitivity to CDK4/6 inhibitors such as abemaciclib (indicated by the yellow arrows) and the antifolate pralatrexate and methotrexate (indicated by the green arrows) compared with the CDKN2A/B/MTAP wild-type lines (NMFH-1 and HFF). Selective sensitivity to PLK-1 inhibitors was detected in WCM197 and OH931 cells compared with the NMFH-1 and the control line HFF, shown in orange arrows. B, Graphs show the response of the sarcoma cells to each compound in the library as area under the curve (AUC) compared with the benign human foreskin fibroblast line (HFF) as a normal control. C and D, Ex vivo drug validation of the two PLK-1 inhibitors (volasertib and rigosertib) confirms high sensitivity for WCM197 and OH931 cells. E and F, WCM197 3D sarcospheres are completely inhibited in growth under volasertib treatment and show cell death indicated by cleaved caspase-3, PARP, and cleaved PARP. Cell death was monitored with cleaved caspase- 3 and cleaved PARP over 96 hours with a peak at 24 to 72 hours.

inhibitors (31). Thus, careful dosing should be considered. As we showed here, a once a week scheduling was well tolerated in mice. Clinical trial data show a good tolerance of these inhibitors when giving them once every 3 weeks as infusion (32). Because sarcomas are such a rare disease, the ability to identify and validate targeted therapies for patients with these tumors after they have failed standard-of-care therapy lags behind that of other cancer types for which there is significantly greater patient volume. By utilizing functional testing of 
A

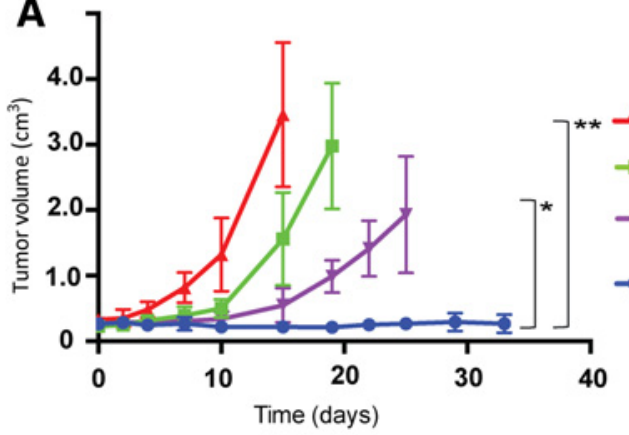

C

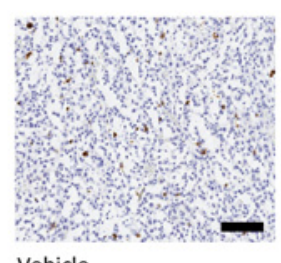

Vehicle

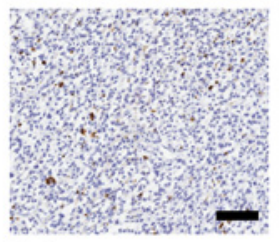

Pralatrexate

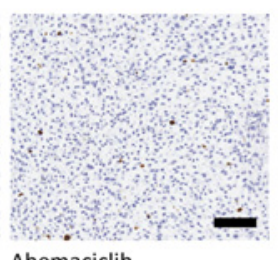

Abemaciclib

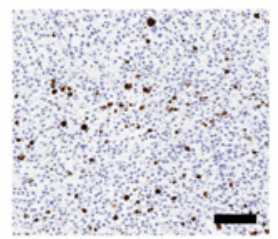

Abemaciclib/Pralatrexate

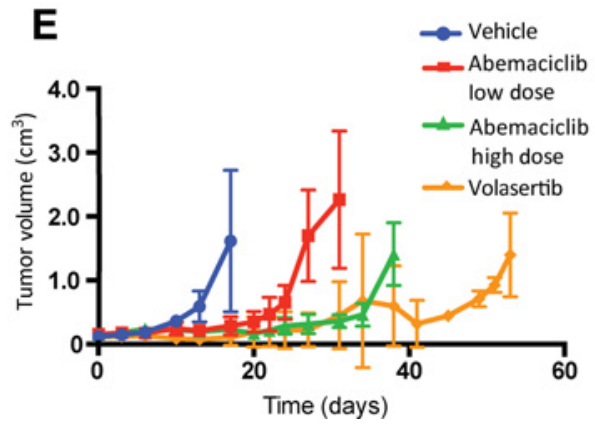

B

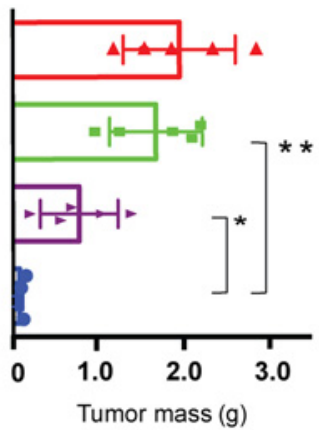

D

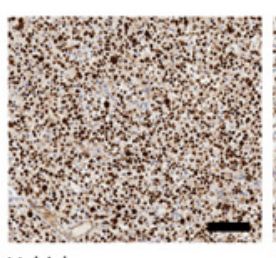

Vehicle

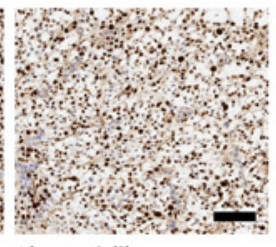

Abemaciclib

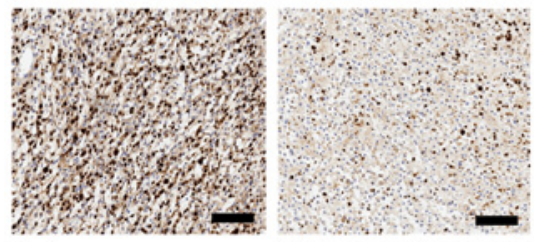

Pralatrexate

Abemaciclib/Pralatrexate
F

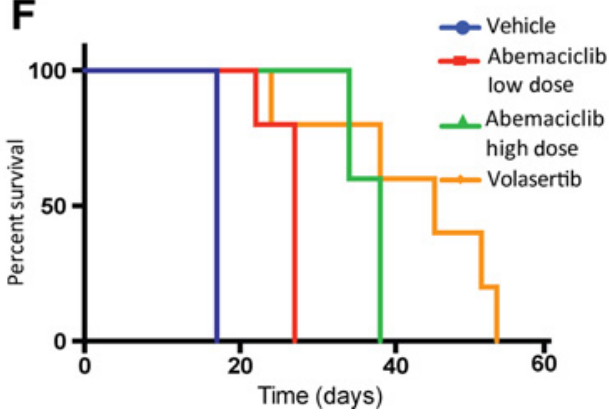

Figure 4.

A and $\mathbf{B}$, In vivo validation of drug sensitivity. Patient-derived xenografts from WCM197 were treated with abemaciclib monotherapy, pralatrexate monotherapy, and the combination of these two drugs versus a vehicle. A significant effect upon tumor growth was observed with the combination when compared with monotherapy with pralatrexate (two-way ANOVA: ${ }^{*}, P=0.0068$ ) and abemaciclib (two-way ANOVA: ${ }^{* *}, P=0.0042$ ). Similarly, tumor mass at endpoint was significantly reduced in the mice treated with combinations of abemaciclib and pralatrexate compared with the monotherapy with pralatrexate (Student $t$ test: $\left.{ }^{*}, P=0.0123\right)$ and abemaciclib (Student $t$ test: ${ }^{*}, P=0.0201$ ). $\mathbf{C}$ and $\mathbf{D}$, Representative images of immunohistochemical stains against cleaved caspase-3 (C) and the proliferation marker Ki67 shows highest cell death and lowest proliferation in the drug combination (D), compared with either drug as single treatment, scale $200 \mu \mathrm{m}$. $\mathbf{E}$ and $\mathbf{F}$, Due to the low toxicity profile of abemaciclib, we tested different concentrations as a monotherapy in patient-derived xenografts from WCM197. A higher daily dose of abemaciclib was beneficial and showed greater tumor growth inhibition, and prolonged survival compared with the lower dose. No weight loss was observed in these animals. Interestingly, using volasertib once a week also extended the survival of these mice up to 55 days.

patient-derived models to complement comprehensive genomic profiling demonstrated the potential of function precision oncology for patients with rare tumor types.

Moving forward, the precision medicine community needs to work with regulatory bodies to develop mechanisms for implementing patient-specific therapeutic strategies for these underserved patients, who are in need of therapeutic options. Building off previous work by our group and others examining common tumor types for which precision medicine approaches are able to identify effective therapeutics, many of which had already been validated through clinical trials (e.g., EGFR inhibitor therapy for KRAS wild-type colon cancer), we demonstrate that the precision medicine approach can be effectively implemented even for patients with rare tumor types. The next step will be to develop a mechanism through which these individualized approaches can be validated in the clinic so that they can be utilized for the benefit of patients with rare diseases for which traditional large scale therapeutic trials are not possible. The current clinical trial formats are designed to validate drugs and drug combinations in the context of larger populations. Here, we extend the potential of precision medicine approaches for patients with rare tumor types, 
providing experimental support for the idea that functional modeling can supplement large genomic data and be leveraged to improve patient treatment options by focusing on tumor-specific sensitivities. The crucial next step will be to identify a mechanism through which functional precision medicine approaches can be brought to the clinic without endangering the patients whom they seek to help.

\section{Authors' Disclosures}

C. Grandori reports other support from SEngine Precision Medicine outside the submitted work, as well as a patent pending to SEngine Precision Medicine. B.D. Hopkins reports grants and other support from Faeth Therapeutics outside the submitted work. No disclosures were reported by the other authors.

\section{Authors' Contributions}

C. Pauli: Conceptualization, resources, data curation, formal analysis, validation, investigation, visualization, methodology, writing-original draft, writing-review and editing. L. De Boni: Data curation, formal analysis, methodology, writing-review and editing. J.E. Pauwels: Data curation, formal analysis, validation, methodology. Y. Chen: Data curation, validation. L. Planas-Paz: Data curation, validation, writing-review and editing. R. Shaw: Data curation, methodology, writing-review and editing. B.M. Emerling: Conceptualization, data curation, methodology, writing-review and editing. C. Grandori: Conceptualization, data curation, formal analysis, validation, visualization, methodology, writing-original draft, writingreview and editing. B.D. Hopkins: Conceptualization, resources, data curation, formal analysis, supervision, funding acquisition, validation, writing-original draft, writing-review and editing. M.A. Rubin: Conceptualization, resources, supervision, funding acquisition, writing-original draft, writing-review and editing.

\section{Acknowledgments}

We would like to thank the patients for entering the precision medicine program and for consenting to our research activities at Weill Cornell Medicine and all the doctors involved.

This work was supported by the Starr Cancer Consortium I7-A771 (M.A. Rubin), R01 CA116337 (M.A. Rubin), Early Detection Research Network US NCI CA111275 (M.A. Rubin), Swiss National Science Foundation P2BEP3_152100 (C. Pauli), OncoSwiss BIL KFS-3259-08-2013 (C. Pauli), NCI U01 CA176303 and U54 CA132381 (C. Grandori), and CA230384-03 from the NCI/NIH (B.D. Hopkins).

The costs of publication of this article were defrayed in part by the payment of page charges. This article must therefore be hereby marked advertisement in accordance with 18 U.S.C. Section 1734 solely to indicate this fact.

Received April 16, 2021; revised September 9, 2021; accepted October 22, 2021; published first November 2, 2021.

\section{References}

1. Fletcher CDM, Bridge JA, Hogendoorn PCW, Mertens F; editors. WHO Classification of Tumors of Soft Tissue and Bone., World Health Organization. Lyon, France: IARC; 2013.

2. Fletcher CD, Gustafson P, Rydholm A, Willen H, Akerman M. Clinicopathologic re-evaluation of 100 malignant fibrous histiocytomas: prognostic relevance of subclassification. J Clin Oncol 2001;19:3045-50.

3. Mentzel T, Calonje E, Wadden C, Camplejohn RS, Beham A, Smith MA, et al. Myxofibrosarcoma. clinicopathologic analysis of 75 cases with emphasis on the low-grade variant. Am J Surg Pathol 1996;20:391-405.

4. Willems SM, Debiec-Rychter M, Szuhai K, Hogendoorn PC, Sciot R. Local recurrence of myxofibrosarcoma is associated with increase in tumour grade and cytogenetic aberrations, suggesting a multistep tumour progression model. Mod Pathol 2006;19:407-16.

5. Sanfilippo R, Miceli R, Grosso F, Fiore M, Puma E, Pennacchioli E, et al. Myxofibrosarcoma: prognostic factors and survival in a series of patients treated at a single institution. Ann Surg Oncol 2011;18:720-5.

6. Huang HY, Lal P, Qin J, Brennan MF, Antonescu CR. Low-grade myxofibrosarcoma: a clinicopathologic analysis of 49 cases treated at a single institution with simultaneous assessment of the efficacy of 3-tier and 4-tier grading systems. Hum Pathol 2004;35:612-21.

7. Barretina J, Taylor BS, Banerji S, Ramos AH, Lagos-Quintana M, DeCarolis PL, et al. Subtype-specific genomic alterations define new targets for soft-tissue sarcoma therapy. Nat Genet 2010;42:715-21.

8. Li CF, Fang FM, Kung HJ, Chen LT, Wang JW, Tsai JW, et al. Downregulated MTAP expression in myxofibrosarcoma: a characterization of inactivating mechanisms, tumor suppressive function, and therapeutic relevance. Oncotarget 2014;5:11428-41.

9. Li CF, Fang FM, Lan J, Wang JW, Kung HJ, Chen LT, et al. AMACR amplification in myxofibrosarcomas: a mechanism of overexpression that promotes cell proliferation with therapeutic relevance. Clin Cancer Res 2014; 20:6141-52.

10. Li CF, Wang JM, Kang HY, Huang CK, Wang JW, Fang FM, et al. Characterization of gene amplification-driven SKP2 overexpression in myxofibrosarcoma: potential implications in tumor progression and therapeutics. Clin Cancer Res 2012;18:1598-610.

11. Taylor B, Olender S, Wilkin TJ, Hammer SM. Advances in antiretroviral therapy. Top Antivir Med 2011;19:69-97.

12. Gibault L, Pérot G, Chibon F, Bonnin S, Lagarde P, Terrier P, et al. New insights in sarcoma oncogenesis: a comprehensive analysis of a large series of $160 \mathrm{soft}$ tissue sarcomas with complex genomics. J Pathol 2011;223:64-71.

13. Ogura K, Hosoda F, Arai Y, Nakamura H, Hama N, Totoki Y, et al. Integrated genetic and epigenetic analysis of myxofibrosarcoma. Nat Commun 2018;9: 2765 .

14. Okada T, Lee AY, Qin LX, Agaram N, Mimae T, Shen Y, et al. Integrin-alpha10 dependency identifies RAC and RICTOR as therapeutic targets in high-grade myxofibrosarcoma. Cancer Discov 2016;6: $1148-65$.

15. The Cancer Genome Atlas Research Network. Comprehensive and integrated genomic characterization of adult soft tissue sarcomas. Cell 2017; 171:950-65.

16. Demetri GD, Heinrich MC, Fletcher JA, Fletcher CDM, Van den Abbeele AD, Corless CL, et al. Molecular target modulation, imaging, and clinical evaluation of gastrointestinal stromal tumor patients treated with sunitinib malate after imatinib failure. Clin Cancer Res 2009;15: 5902-9.

17. Bender J, Anderson B, Bloom DA, Rabah R, McDougall R, Vats P, et al. Refractory and metastatic infantile fibrosarcoma harboring LMNA-NTRK1 fusion shows complete and durable response to crizotinib. Cold Spring Harb Mol Case Stud 2019;5:a003376.

18. Beltran H, Eng K, Mosquera JM, Sigaras A, Romanel A, Rennert H, et al. Wholeexome sequencing of metastatic cancer and biomarkers of treatment response. JAMA Oncol 2015;1:466-74.

19. Prandi D, Baca SC, Romanel A, Barbieri CE, Mosquera JM, Fontugne J, et al. Unraveling the clonal hierarchy of somatic genomic aberrations. Genome Biol 2014;15:439.

20. Kawashima H, Ogose A, Gu W, Nishio J, Kudo N, Kondo N, et al. Establishment and characterization of a novel myxofibrosarcoma cell line. Cancer Genet Cytogenet 2005;161:28-35.

21. Krause AK, Hinrichs SH, Orndal C, DeBoer J, Neff JR, Bridge JA. Characterization of a human myxoid malignant fibrous histiocytoma cell line, OH931. Cancer Genet Cytogenet 1997;94:138-43.

22. Benanti JA, Wang ML, Myers HE, Robinson KL, Grandori C, Galloway DA. Epigenetic down-regulation of ARF expression is a selection step in immortalization of human fibroblasts by c-Myc. Mol Cancer Res 2007;5: 1181-9.

23. Pauli C, Hopkins BD, Prandi D, Shaw R, Fedrizzi T, Sboner A, et al. Personalized in vitro and in vivo cancer models to guide precision medicine. Cancer Discov 2017;7:462-77.

24. Patnaik A, Rosen LS, Tolaney SM, Tolcher AW, Goldman JW, Gandhi L, et al. Efficacy and safety of abemaciclib, an inhibitor of CDK4 and CDK6, for patients with breast cancer, non-small cell lung cancer, and other solid tumors. Cancer Discov 2016;6:740-53.

25. Illei PB, Rusch VW, Zakowski MF, Ladanyi M. Homozygous deletion of CDKN2A and codeletion of the methylthioadenosine phosphorylase gene in the majority of pleural mesotheliomas. Clin Cancer Res 2003; 9:2108-13. 
26. Bertino JR, Lubin M, Johnson-Farley N, Chan WC, Goodell L, Bhagavathi S. Lack of expression of MTAP in uncommon T-cell lymphomas. Clin Lymphoma Myeloma Leuk 2012;12:306-9.

27. von Witzleben A, Goerttler LT, Marienfeld R, Barth H, Lechel A, Mellert K, et al. Preclinical characterization of novel chordoma cell systems and their targeting by pharmocological inhibitors of the CDK4/6 cell-cycle pathway. Cancer Res 2015;75:3823-31.

28. Elvin JA, Gay LM, Ort R, Shuluk J, Long J, Shelley L, et al. Clinical benefit in response to palbociclib treatment in refractory uterine leiomyosarcomas with a common CDKN2A alteration. Oncologist 2017;22:416-21.
29. Laroche-Clary A, Chaire V, Algeo M-P, Derieppe M-A, Loarer FL, Italiano A Combined targeting of MDM2 and CDK4 is synergistic in dedifferentiated liposarcomas. J Hematol Oncol 2017;10:123.

30. Guenther LM, Dharia NV, Ross L, Conway A, Robichaud AL, Catlett JL, et al. A combination CDK4/6 and IGF1R inhibitor strategy for Ewing sarcoma. Clin Cancer Res 2019;25:1343-57.

31. de Carcer G. The mitotic cancer target polo-like kinase 1: oncogene or tumor suppressor? Genes 2019;10:208.

32. Hao Z, Kota V. Volasertib for AML: clinical use and patient consideration. Onco Targets Ther 2015;8:1761-71. 


\section{Molecular Cancer Research}

\section{A Functional Precision Oncology Approach to Identify Treatment Strategies for Myxofibrosarcoma Patients}

Chantal Pauli, Lamberto De Boni, Jonathan E. Pauwels, et al.

Mol Cancer Res Published OnlineFirst November 2, 2021.

Updated version

Supplementary Material
Access the most recent version of this article at: doi:10.1158/1541-7786.MCR-21-0255

Access the most recent supplemental material at: http://mcr.aacrjournals.org/content/suppl/2021/10/27/1541-7786.MCR-21-0255.DC1

E-mail alerts Sign up to receive free email-alerts related to this article or journal.

Reprints and To order reprints of this article or to subscribe to the journal, contact the AACR Publications Subscriptions Department at pubs@aacr.org.

Permissions To request permission to re-use all or part of this article, use this link http://mcr.aacrjournals.org/content/early/2021/12/03/1541-7786.MCR-21-0255. Click on "Request Permissions" which will take you to the Copyright Clearance Center's (CCC)

Rightslink site. 